\title{
Existence and Uniqueness of the Solution for an Integral Equation with Supremum, via $w$-Distances
}

\author{
Veronica Ilea ${ }^{1}$ and Diana Otrocol $2,3, * \mathbb{C}$ \\ 1 Babeş-Bolyai University, Department of Mathematics, 1 M. Kogălniceanu Street, 400084 Cluj-Napoca, \\ Romania; vdarzu@math.ubbcluj.ro \\ 2 Technical University of Cluj-Napoca, Department of Mathematics, 28 Memorandumului Street, \\ 400114 Cluj-Napoca, Romania \\ 3 Tiberiu Popoviciu Institute of Numerical Analysis, Romanian Academy, P.O. Box 68-1, \\ 400110 Cluj-Napoca, Romania \\ * Correspondence: dotrocol@ictp.acad.ro or Diana.Otrocol@math.utcluj.ro
}

Received: 2 September 2020; Accepted: 18 September 2020; Published: 20 September 2020

\begin{abstract}
Following the idea of T. Wongyat and W. Sintunavarat, we obtain some existence and uniqueness results for the solution of an integral equation with supremum. The paper ends with the study of Gronwall-type theorems, comparison theorems and a result regarding a Ulam-Hyers stability result for the corresponding fixed point problem.
\end{abstract}

Keywords: $w$-distance; integral equation with supremum; abstract Gronwall theorem; weakly Picard operator; Ulam-Hyers stability

\section{Introduction}

The object of investigation of this paper is the qualitative theory of integral equations with supremum. These equations arise naturally when solving real-world problems, for example in the study of systems with automatic regulation and automatic control, problems in control theory. These types of equations are characterized by the fact that the maximum values of some regulated state parameters depend on certain time intervals, see for example [1] and the references therein. Recently, the interest in differential equations with supremum has an intensive development (see [2-4]). The aim of this paper focuses on two aspects: one is to prove existence and uniqueness results using $w$-weak generalized contractions theorem; the other is to prove a Gronwall-type theorem and comparison theorems. Using this theory symmetry is important in determining the qualitative properties of the solution of the integral equation.

We consider the following class of integral equation with supremum

$$
x(t)=\varphi(t)+\int_{\alpha}^{t} f\left(t, s, x(s) \sup _{\theta \in[\alpha, s]} x(\theta)\right) d s, t \in[\alpha, \beta]
$$

with $\alpha, \beta$ real and $\alpha<\beta$, the functions $\varphi \in C([\alpha, \beta], \mathbb{R}), f \in C\left([\alpha, \beta] \times[\alpha, \beta] \times \mathbb{R}^{2}, \mathbb{R}\right)$ are given. To prove our results, we shall use the $w$-weak generalized contractions theorem due to T. Wongyat and W. Sintunavarat [5] and we obtain an existence and uniqueness result for the solutions of this equation.

\section{Preliminaries}

We consider $(\mathcal{T}, d)$ a metric space. In the sequel, we will use the following definitions and theorems, for details, see $[5,6]$. 
Definition 1. ([6]) Let $(\mathcal{T}, d)$ a metric space and a function $q: \mathcal{T} \times \mathcal{T} \rightarrow[0, \infty)$. We say that $q$ is a $w$-distance on $\mathcal{T}$, if the below conditions hold, for all $x, y, z \in \mathcal{T}$ :

(1) $q(x, y) \leq q(x, z)+q(z, y)$;

(2) $\quad q(x, \cdot): \mathcal{T} \rightarrow[0, \infty)$ is lower semicontinuous;

(3) for each $\varepsilon>0$, there exists $\delta>0$ such that $q(x, y) \leq \delta$ and $q(x, z) \leq \delta$ imply $d(y, z) \leq \varepsilon$.

We recall that each metric on the nonempty set $\mathcal{T}$ is a $w$-distance on $\mathcal{T}$.

Definition 2 ([5]). We say that the function $\psi: \mathcal{T} \times \mathcal{T} \rightarrow[0, \infty)$ is a $w^{0}$-distance on $\mathcal{T}$, if it is a $w$-distance on $\mathcal{T}$ with $q(x, x)=0$, for all $x \in \mathcal{T}$.

Definition 3 ([5]). We say that the function $\psi:[0, \infty) \rightarrow[0, \infty)$ is an altering distance function, if the below assertions hold:

(1) The function $\psi$ is continuous and nondecreasing;

(2) $\psi(t)$ is zero if and only if $t=0$.

Definition 4 ([5]). Let $(\mathcal{T}, d)$ be a metric space. We say that a $w$-distance $q$ is a ceiling distance of $d$ if and only if $q(x, y) \geq d(x, y)$, for all $x, y \in \mathcal{T}$.

Definition 5 ([5]). We consider $q$ a $w$-distance on the metric space $(\mathcal{T}, d)$, the altering distance function $\psi:[0, \infty) \rightarrow[0, \infty)$, and the continuous function $\phi:[0, \infty) \rightarrow[0, \infty)$ with $\phi(t)$ is zero if and only if $t=0$. If the below inequality holds we say that the operator $A: \mathcal{T} \rightarrow \mathcal{T}$ is a w-generalized weak contraction mapping

$$
\psi(q(A(x), A(y))) \leq \psi(m(x, y)-\phi(q(x, y))), \text { for all } x, y \in \mathcal{T},
$$

where

$$
m(x, y):=\max \left\{q(x, y), \frac{q(x, A(y))+q(A(x), y)}{2}\right\} .
$$

If $q=d$, then we say that $A$ is a generalized weak contraction mapping.

Now we consider $(\mathcal{T}, d)$ a complete metric space. The following fixed point result of the equation $A(x)=x, x \in \mathcal{T}$ via $w$-distances represents the motivation of our work.

Theorem 1 ([5]). We consider $q: \mathcal{T} \times \mathcal{T} \rightarrow[0, \infty)$ a continuous $w$-distance on $\mathcal{T}$ and a ceiling distance of $d$, the altering distance function $\psi:[0, \infty) \rightarrow[0, \infty)$, and the continuous function $\phi:[0, \infty) \rightarrow[0, \infty)$ with $\phi(t)$ is zero if and only if $=0$. Let $A: \mathcal{T} \rightarrow \mathcal{T}$ a continuous operator such that

$$
\psi(q(A(x), A(y))) \leq \psi(m(x, y)-\phi(q(x, y))), \text { for all } x, y \in \mathcal{T} .
$$

Then, $A$ has a unique fixed point in $\mathcal{T}$ and the sequence of successive approximations $\left\{x_{n}\right\}_{n \in \mathbb{N}}$, defined by $x_{n}=A^{n}\left(x_{0}\right)$, for each $x_{0} \in \mathcal{T}$, for all $n \in \mathbb{N}$, converges to the unique fixed point of $A$.

For other fixed points results obtained employing the theory of $w$-distance, the reader is referred to $[5,7-11]$.

In this paper, we emphasize some connection between $w$-generalized weak contraction mapping and the Picard operator theory.

In the sequel, we recall the following results (see [12-14]).

Let $(\mathcal{T}, d)$ be a metric space. We say that the operator $A: \mathcal{T} \rightarrow \mathcal{T}$ is weakly a Picard operator (WPO) if the successive approximations sequence $\left\{A^{n}(x)\right\}_{n \in \mathbb{N}}$, converges for all $x \in \mathcal{T}$ and its limit (which generally depend on $x$ ) is a fixed point of $A$. If an operator $A$ is WPO with $F_{A}=\left\{x^{*}\right\}$, then, we say that the operator $A$ is a Picard operator (PO). 
If $A: \mathcal{T} \rightarrow \mathcal{T}$ is a WPO, we can define the operator $A^{\infty}: \mathcal{T} \rightarrow F_{A}$, by $A^{\infty}(x):=\lim _{n \rightarrow+\infty} A^{n}(x)$

Definition 6. Let $A$ be a weakly Picard operator and $c>0$. We say that the operator $A$ is a c-weakly Picard operator if

$$
d\left(x, A^{\infty}(x)\right) \leq c d(x, A(x)), \text { for all } x \in \mathcal{T} .
$$

If $\mathcal{T}$ is a nonempty set, then $(\mathcal{T}, d, \leq)$ is an ordered metric space, where $\leq$ is a partial order relation on $\mathcal{T}$.

Now we present some properties regarding WPOs and POs.

Theorem 2 ([12]). (Characterization theorem) Let $(\mathcal{T}, d)$ be a metric space. The operator $A: \mathcal{T} \rightarrow \mathcal{T}$ is WPO if there exists a partition of $\mathcal{T}, \mathcal{T}=\underset{\lambda \in \Lambda}{\cup} X_{\lambda}$, such that

(a) $\mathcal{T}_{\lambda} \in I(A)$, for all $\lambda \in \Lambda$;

(b) $\left.A\right|_{\mathcal{T}_{\lambda}}: \mathcal{T}_{\lambda} \rightarrow \mathcal{T}_{\lambda}$ is $P O$, for all $\lambda \in \Lambda$.

Theorem 3 ([13]). (Abstract Gronwall Theorem) Let $(\mathcal{T}, d, \leq)$ be an ordered metric space and we consider the operator $A: \mathcal{T} \rightarrow \mathcal{T}$. We suppose

(i) The operator $A$ is increasing with respect to $\leq$;

(ii) $A$ is a Picard operator with $F_{A}=\left\{x^{*}\right\}$.

Then the below conclusions hold:

(i) for $x \in \mathcal{T}$, $x \leq A(x) \Rightarrow x \leq x^{*}$,

(ii) for $x \in \mathcal{T}, x \geq A(x) \Rightarrow x \geq x^{*}$.

Theorem 4 ([13]). (Abstract Comparison Lemma) Let $(\mathcal{T}, d, \leq)$ be an ordered metric space and we consider the operators $A, B, C: \mathcal{T} \rightarrow \mathcal{T}$ with the properties:

(i) $A \leq B \leq C$;

(ii) $A, B, C$ are WPOs;

(iii) $B$ is an increasing operator.

Then, for $x, y, z \in \mathcal{T}, x \leq y \leq z \Rightarrow A^{\infty}(x) \leq B^{\infty}(y) \leq C^{\infty}(z)$.

We present now the concept of Hyers-Ulam stability in the setting of metric spaces given by I.A. Rus in [15].

Definition 7. Let $(\mathcal{T}, d)$ be a metric space and we consider the operator $A: \mathcal{T} \rightarrow \mathcal{T}$. Then, we say that the fixed point equation

$$
x=A(x)
$$

is Ulam-Hyers stable if there exists $c_{A} \in \mathbb{R}_{+}^{*}$ such that: for any $\varepsilon>0$ and for each solution $y^{*} \in \mathcal{T}$ of (5), i.e., $d\left(y^{*}, A\left(y^{*}\right)\right) \leq \varepsilon$, there exists a solution $x^{*}$ of (5) such that

$$
d\left(y^{*}, x^{*}\right) \leq c_{A} \varepsilon .
$$

We recall the following abstract result of the Ulam-Hyers stability of the fixed point Equation (5).

Theorem 5. (Ulam-Hyers stability, [15]) Let $(\mathcal{T}, d)$ be a metric space. Suppose that $A: \mathcal{T} \rightarrow \mathcal{T}$ is a c-Picard operator. Then, Equation (5) is Ulam-Hyers stable.

For more results regarding WPOs and POs, see [3,4,14-16]. 


\section{Main Result}

Let the operator $A: C([\alpha, \beta], \mathbb{R}) \rightarrow C([\alpha, \beta], \mathbb{R})$ expressed by

$$
A(x)(t):=\varphi(t)+\int_{\alpha}^{t} f\left(t, s, x(s), \sup _{\theta \in[\alpha, s]} x(\theta)\right) d s,
$$

where $f \in C\left([\alpha, \beta] \times[\alpha, \beta] \times \mathbb{R}^{2}, \mathbb{R}\right), x \in C([\alpha, \beta], \mathbb{R})$ and $t \in[\alpha, \beta]$.

Our first result is the following theorem.

Theorem 6. We consider the integral Equation (1) with $\alpha, \beta$ real and $\alpha<\beta$, the functions $\varphi \in$ $C([\alpha, \beta], \mathbb{R}), f \in C\left([\alpha, \beta] \times[\alpha, \beta] \times \mathbb{R}^{2}, \mathbb{R}\right)$ are given. We assume the following:

(i) The operator $A: C([\alpha, \beta], \mathbb{R}) \rightarrow C([\alpha, \beta], \mathbb{R})$ defined by (6) is continuous;

(ii) The altering distance function $\psi:[0, \infty) \rightarrow[0, \infty)$ satisfies $\psi(t)<t$, for all $t>0$, and the continuous function $\phi:[0, \infty) \rightarrow[0, \infty)$ satisfies $\phi(t)$ is zero if and only if $t=0$;

(iii) The below inequality holds

$$
\begin{aligned}
& \left|f\left(t, s, x(s), \sup _{\theta \in[\alpha, s]} x(\theta)\right)\right|+\left|f\left(t, s, y(s), \sup _{\theta \in[\alpha, s]} y(\theta)\right)\right| \\
& \leq \frac{\psi\left(|x(s)|+|y(s)|+\left|\sup _{\theta \in[\alpha, s]} x(\theta)\right|+\left|\sup _{\theta \in[\alpha, s]} y(\theta)\right|\right)}{\beta-\alpha} \\
& -\frac{\phi\left(\sup _{l \in[\alpha, \beta]}|x(l)|+\sup _{l \in[\alpha, \beta]}|y(l)|+\sup _{l \in[\alpha, \beta]}\left|\sup _{\theta \in[\alpha, l]} x(\theta)\right|+\sup _{l \in[\alpha, \beta]}\left|\sup _{\theta \in[\alpha, l]} y(\theta)\right|\right)}{\beta-\alpha} \\
& -\frac{2|\varphi(t)|}{\beta-\alpha}, \text { for all } x, y \in C([\alpha, \beta], \mathbb{R}), t, s \in[\alpha, \beta] .
\end{aligned}
$$

Then the integral equation with supremum (1) has a unique solution and the sequence of successive approximations $\left\{x_{n}\right\}_{n \in \mathbb{N}}$, defined by $x_{n}=A^{n}\left(x_{0}\right)$, for each $x_{0} \in C([\alpha, \beta], \mathbb{R})$, for all $n \in \mathbb{N}$, converges to the unique solution of Equation (1).

Proof. Let $\mathcal{T}=C([\alpha, \beta], \mathbb{R})$ and we consider the metric $d: \mathcal{T} \times \mathcal{T} \rightarrow[0, \infty)$ defined as below

$$
d(x, y):=\sup _{t \in[\alpha, \beta]}|x(t)-y(t)|, \text { for all } x, y \in C([\alpha, \beta], \mathbb{R}) .
$$

It is clear that $(\mathcal{T}, d)$ is a complete metric space. We consider the function $q: C([\alpha, \beta], \mathbb{R}) \times$ $C([\alpha, \beta], \mathbb{R}) \rightarrow[0, \infty)$ defined by:

$$
q(x, y):=\sup _{t \in[\alpha, \beta]}|x(t)|+\sup _{t \in[\alpha, \beta]}|y(t)|, \text { for all } x, y \in C([\alpha, \beta], \mathbb{R}) .
$$

We get that $q$ is a $w$-distance on $\mathcal{T}$ and also a ceiling distance of $d$. 
We will show that $A$ satisfies the contraction condition (4).

$$
\begin{aligned}
& |A(x)(t)|+|A(y)(t)| \\
& =\left|\varphi(t)+\int_{\alpha}^{t} f\left(t, s, x(s), \sup _{\theta \in[\alpha, s]} x(\theta)\right) d s\right|+\left|\varphi(t)+\int_{\alpha}^{t} f\left(t, s, y(s), \sup _{\theta \in[\alpha, s]} y(\theta)\right) d s\right| \\
& \leq|\varphi(t)|+\left|\int_{\alpha}^{t} f\left(t, s, x(s), \sup _{\theta \in[\alpha, s]} x(\theta)\right) d s\right|+|\varphi(t)| \\
& +\left|\int_{\alpha}^{t} f\left(t, s, y(s), \sup _{\theta \in[\alpha, s]} y(\theta)\right) d s\right| \\
& \leq 2|\varphi(t)|+\int_{\alpha}^{t}\left(\left|f\left(t, s, x(s), \sup _{\theta \in[\alpha, s]} x(\theta)\right)\right|+\left|f\left(t, s, y(s), \sup _{\theta \in[\alpha, s]} y(\theta)\right)\right|\right) d s \\
& \leq 2|\varphi(t)|+\frac{1}{\beta-\alpha} \int_{\alpha}^{t}\left[\psi\left(|x(s)|+|y(s)|+|\sup x(\theta)|+\left|\sup _{\theta \in[\alpha, s]} y(\theta)\right|\right)\right. \\
& \left.-\phi\left(\sup _{l \in[\alpha, \beta]}|x(l)|+\sup _{l \in[\alpha, \beta]}|y(l)|+\sup _{l \in[\alpha, \beta]}\left|\sup _{\theta \in[\alpha, l]} x(\theta)\right|+\sup _{l \in[\alpha, \beta]}\left|\sup _{\theta \in[\alpha, l]} y(\theta)\right|\right)-2|\varphi(t)|\right] d s \\
& \leq \psi(q(x, y)-\phi(q(x, y))) .
\end{aligned}
$$

We obtain that

$$
\sup _{t \in[\alpha, \beta]}|A x(t)|+\sup _{t \in[\alpha, \beta]}|A y(t)| \leq \psi(q(x, y)-\phi(q(x, y)))
$$

and using (8) we get

$$
q(A x, A y) \leq \psi(q(x, y)-\phi(q(x, y))), \text { for all } x, y \in \mathcal{T}
$$

Hence we have

$$
\psi(q(A x, A y)) \leq q(A x, A y) \leq \psi(q(x, y))-\phi(q(x, y)), \text { for all } x, y \in \mathcal{T}
$$

Therefore the condition (4) holds and thus we may conclude that $A$ has a unique fixed point. So there exists a unique solution for the integral equation with supremum (1).

From the above theorem, the operator $A$ defined in (6) is a PO. Now we establish a Gronwall-type theorem for Equation (1).

Theorem 7. We consider the integral Equation (1) with $\alpha, \beta$ real, $\alpha<\beta$, and the functions $\varphi \in$ $C([\alpha, \beta], \mathbb{R}), f \in C([\alpha, \beta] \times[\alpha, \beta] \times \mathbb{R}, \mathbb{R})$ are given. We assume that the conditions (i)-(iii) from Theorem 6 hold. Furthermore, we suppose that

(iv) $f(t, s, \cdot): \mathbb{R} \rightarrow \mathbb{R}$ is an increasing function with respect to the last argument, for all $t, s \in[\alpha, \beta]$.

Let $x^{*} \in C([\alpha, \beta], \mathbb{R})$ be the unique solution of the integral Equation (1). Then, the following conditions are satisfied:

(1) for all $x \in C([\alpha, \beta], \mathbb{R})$ with

$$
x(t) \leq \varphi(t)+\int_{\alpha}^{t} f\left(t, s, \sup _{\theta \in[\alpha, s]} x(\theta)\right) d s, t \in[\alpha, \beta]
$$

we have $x \leq x^{*}$; 
(2) for all $x \in C([\alpha, \beta], \mathbb{R})$ with

$$
x(t) \geq \varphi(t)+\int_{\alpha}^{t} f\left(t, s, \sup _{\theta \in[\alpha, s]} x(\theta)\right) d s, t \in[\alpha, \beta]
$$

we have $x \geq x^{*}$.

Proof. From (iv), we have that the operator $A$ defined in (6) is increasing with respect to the partial order.

By the proof of Theorem 6, it follows that A is a Picard operator. The conclusion of the theorem follows from Theorem 3.

We establish now a comparison theorem for Equation (1), using Theorem 4.

Theorem 8. We consider the integral Equation (1) with $\alpha, \beta$ real, $\alpha<\beta$, and we suppose that $\varphi_{i} \in C([\alpha, \beta], \mathbb{R})$ and $f_{i} \in C([\alpha, \beta] \times[\alpha, \beta] \times \mathbb{R}, \mathbb{R}), i=1,2,3$ are given. We assume that the conditions (i)-(iii) from Theorem 6 hold. Furthermore, we suppose that

(i) $\varphi_{1} \leq \varphi_{2} \leq \varphi_{3}, f_{1} \leq f_{2} \leq f_{3}$;

(ii) $\varphi_{2}, f_{2}$ are increasing.

Let $x_{i} \in C([\alpha, \beta], \mathbb{R})$ be a solution of the equation

$$
x_{i}(t)=\varphi_{i}(t)+\int_{\alpha}^{t} f_{i}\left(t, s, \sup _{\theta \in[\alpha, s]} x(\theta)\right) d s, t \in[\alpha, \beta], i=1,2,3 .
$$

If $x_{1}(\alpha) \leq x_{2}(\alpha) \leq x_{3}(\alpha)$, then $x_{1} \leq x_{2} \leq x_{3}$.

Proof. The proof follows from the Theorem 4.

Now we prove a Ulam-Hyers stability result for the integral Equation (1).

Theorem 9. We consider the integral equation with supremum (1) and we suppose that all the conditions of Theorem 6 are satisfied. Then, the integral Equation (1) is Ulam-Hyers stable.

Proof. Applying Theorem 6 and Theorem 5 we get the conclusion of the theorem.

\section{Conclusions}

The purpose of this paper is to establish some fixed point results for generalized contraction operators with respect to $w$-distances. The operators considered here contain a supremum over a certain time interval. Section 3 begins with an existence and uniqueness theorem proved using the method of $w$-distances. Adding to the hypotheses that sustain the existence and uniqueness of the solution, the fact that $f$ is an increasing function, we obtain Gronwall-type and comparison theorems. In the last part of the paper we study the Ulam-Hyers stability using Picard operators techniques. We define a fixed point equation from the integral equation with supremum. If the defined operator is $c$-weakly Picard we have Ulam-Hyers stability of the corresponding fixed point problem.

Author Contributions: Conceptualization, V.I. and D.O.; methodology, V.I. and D.O.; validation, V.I. and D.O.; formal analysis, V.I. and D.O.; investigation, V.I. and D.O.; resources, V.I. and D.O.; writing-original draft preparation, V.I. and D.O.; writing-review and editing, V.I. and D.O.; visualization, V.I. and D.O.; supervision, V.I. and D.O.; project administration, V.I. and D.O. All authors have read and agreed to the published version of the manuscript.

Funding: This research received no external funding.

Conflicts of Interest: The authors declare no conflict of interest. 


\section{References}

1. Popov, E. Automatic Regulation and Control; Moscow, Russia, 1966. (In Russian)

2. Bainov, D.D.; Hristova, S. Differential Equations with Maxima; Chapman \& Hall/CRC Pure and Applied Mathematics: Boca Raton, FL, USA, 2011.

3. Otrocol, D. Hybrid differential equations with maxima via Picard operators theory. Stud. Univ. Babeş-Bolyai Math. 2016, 61, 421-428.

4. Otrocol, D.; Rus, I.A. Functional-differential equations with "maxima" via weakly Picard operators theory. Bull. Math. Soc. Sci. Math. Roum. 2008, 51, 253-261.

5. Wongyat, T.; Sintunavarat, W. The existence and uniqueness of the solution for nonlinear Fredholm and Volterra integral equations together with nonlinear fraction differential equations via $w$-distances. Adv. Diff. Equ. 2017, 2017, 211. [CrossRef]

6. Kada, O.; Suzuki, T.; Takahashi, W. Nonconvex minimization theorems and fixed point theorems in complete metric spaces. Math. Jpn. 1996, 44, 381-391.

7. Aguirre Salazar, L.; Reich, S. A remark on weakly contractive mappings. J. Nonlinear Conv. Anal. 2015, 16, 767-773.

8. Dobriţoiu, M. An application of the $w$-weak generalized contractions theorem. J. Fixed Point Theory Appl. 2019, 21, 93. [CrossRef]

9. Suzuki, T.; Takahashi, W. Fixed points theorems and characterizations of metric completeness. Topol. Methods Nonlinear Anal. J. Juliusz Schauder Cent. 1996, 8, 371-382. [CrossRef]

10. Takahashi, W.; Wong, N.C.; Yao, J.C. Fixed point theorems for general contractive mappings with $w$-distances in metric spaces. J. Nonlinear Conv. Anal. 2013, 14, 637-648.

11. Wongyat, T.; Sintunavarat, W. On new existence and uniqueness results concerning solutions to nonlinear Fredholm integral equations via w-distances and weak altering distance functions. J. Fixed Point Theory Appl. 2019, 21, 7. [CrossRef]

12. Rus, I.A. Generalized Contractions and Applications; Cluj University Press: Cluj-Napoca, Romania, 2001.

13. Rus, I.A. Picard operators and applications. Sci. Math. Jpn. 2003, 58, 191-219.

14. Rus, I.A. Fixed points, upper and lower fixed points: Abstract Gronwall lemmas. Carpathian J. Math. 2004, 20, 125-134.

15. Rus, I.A. Remarks on Ulam stability of the operatorial equations. Fixed Point Theory 2009, 10, 305-320.

16. Ilea, V.A.; Otrocol, D. An application of the Picard operator technique to functional integral equations. J. Nonlinear Conv. Anal. 2017, 18, 405-413.

(C) 2020 by the authors. Licensee MDPI, Basel, Switzerland. This article is an open access article distributed under the terms and conditions of the Creative Commons Attribution (CC BY) license (http://creativecommons.org/licenses/by/4.0/). 